\title{
Incorporating Target Registration Error Into Robotic Bone Milling
}

\author{
Michael A. Siebold ${ }^{* a}$, Neal P. Dillon ${ }^{\mathrm{b}}$, Robert J. Webster III ${ }^{\mathrm{a}, \mathrm{b}}$, and J. Michael Fitzpatrick ${ }^{\mathrm{a}}$ \\ ${ }^{\mathrm{a}}$ Electrical Engineering and Computer Science, ${ }^{\mathrm{b}}$ Mechanical Engineering \\ Vanderbilt University, Nashville, TN 37235, USA
}

\begin{abstract}
Robots have been shown to be useful in assisting surgeons in a variety of bone drilling and milling procedures. Examples include commercial systems for joint repair or replacement surgeries, with in vitro feasibility recently shown for mastoidectomy. Typically, the robot is guided along a path planned on a CT image that has been registered to the physical anatomy in the operating room, which is in turn registered to the robot. The registrations often take advantage of the high accuracy of fiducial registration, but, because no real-world registration is perfect, the drill guided by the robot will inevitably deviate from its planned path. The extent of the deviation can vary from point to point along the path because of the spatial variation of target registration error. The allowable deviation can also vary spatially based on the necessary safety margin between the drill tip and various nearby anatomical structures along the path. Knowledge of the expected spatial distribution of registration error can be obtained from theoretical models or experimental measurements and used to modify the planned path. The objective of such modifications is to achieve desired probabilities for sparing specified structures. This approach has previously been studied for drilling straight holes but has not yet been generalized to milling procedures, such as mastoidectomy, in which cavities of more general shapes must be created. In this work, we present a general method for altering any path to achieve specified probabilities for any spatial arrangement of structures to be protected. We validate the method via numerical simulations in the context of mastoidectomy.
\end{abstract}

Keywords: surgery, robot, registration, TRE, path planning, mastoidectomy

\section{INTRODUCTION}

Bone milling (i.e. using a surgical drill to create a cavity in the bone) was one of the first procedures targeted in surgical robotics research ${ }^{1,2}$, and several companies have successfully implemented robotic systems for joint resurfacing and arthroplasty. One example is the Robodoc system (Curexo, Inc.), which autonomously mills a desired volume of bone based on a pre-operative plan. Another is the RIO system (Mako Surgical, Inc.), which uses a cooperative control approach in which both the surgeon and the robot simultaneously hold the surgical drill, with the robot imposing boundaries called "virtual fixtures" ${ }^{3}$ that prevent the surgeon from accidentally milling unintended bone regions. Another company that has developed a clinical system is URS Ortho GMBH \& Co. KG with their CASPAR platform.

Recently, several research groups have used conceptually similar robots for bone removal in skull base surgery ${ }^{5-8}$. One procedure considered is robotic mastoidectomy, which is the removal of all or part of the mastoid region of the temporal bone to gain access to the middle and inner ear for various surgical procedures. While the general concept in mastoidectomy is similar to orthopedic bone milling (i.e. accurately removing a desired volume of bone), this anatomical region presents additional challenges that require high accuracy. Many critical structures are embedded within and around the targeted bone (e.g. Facial Nerve, Chorda Tympani, etc., see Figure 1), and the surgical drill must pass in close proximity to them. Thus, high accuracy is required to safely remove the required volume of bone ${ }^{9}$.

One thing that is common to all image-guided bone milling procedures is the need to accurately register preoperative images to the patient and the robot. Since this registration process will never be perfectly accurate, there is a risk that the drill bit will accidentally deviate outside the planned milling volume. There is currently no way to guarantee that the probability of doing so is below a specific desired threshold.

The purpose of this paper is to address this risk by using Target Registration Error theory to establish a spatially varying, minimally thick, safety margin around any critical structures relevant to the procedure, as well as around the boundary of the planned milling volume itself. This approach will enable the surgeon to quantify the risk tolerance for each structure (e.g. the surgeon might do this by stating something like: "I am comfortable with the robot accidentally drilling a tiny bit

michael.a.siebold@vanderbilt.edu; http://research.vuse.vanderbilt.edu/MEDLab/

Medical Imaging 2015: Image-Guided Procedures, Robotic Interventions, and Modeling,

edited by Ziv R. Yaniv, Robert J. Webster III, Proc. of SPIE Vol. 9415, 94150R

(C) 2015 SPIE $\cdot$ CCC code: $1605-7422 / 15 / \$ 18 \cdot$ doi: $10.1117 / 12.2082340$

Proc. of SPIE Vol. 9415 94150R-1 
more bone than planned in 1 out of 100 cases in a knee implant", or "The robot hitting the Facial nerve in 1 out of 1000 cases is acceptable."). Our algorithm will then enable the robot to mill out as much bone as is possible, while satisfying the surgeon's probability specifications.
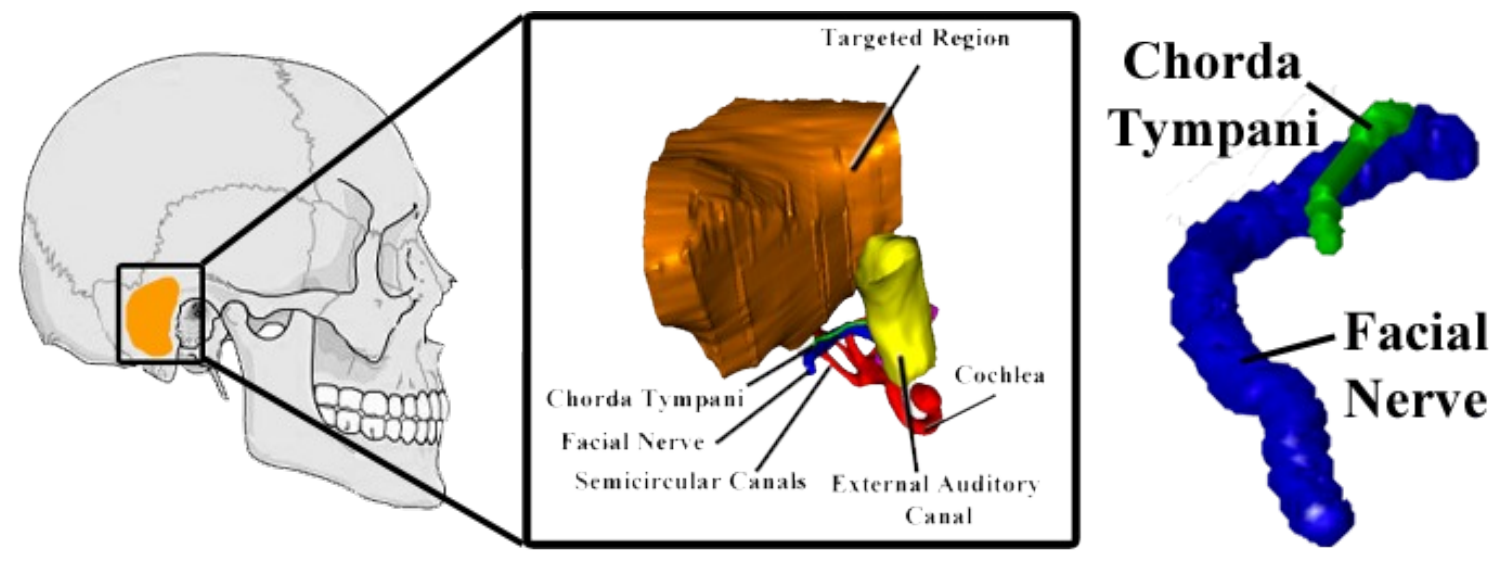

Figure 1: While our algorithm applies to any bone milling procedure, mastoidectomy is of particular interest and is the context in which we test the algorithm in this paper. Mastoidectomy involves a Targeted Region of bone to be removed, which is in close proximity to delicate and vital structures such as the Facial Nerve that must not be damaged by the drill bit. Throughout the remainder of the paper we will often focus on the Facial Nerve and Chorda Tympani as examples of structures that must be preserved with surgeon-specified probability.

Our algorithm assumes that Target Registration Error (TRE) can be calculated. In this paper we assume that registration is accomplished via fiducial markers, in which case TRE formulas are available in ${ }^{10}$. We also assume that a segmented medical image is available with required (potentially spatially varying) surgeon-specified probability thresholds defined around critical structures and along the boundary of the Targeted Region to be milled. This algorithm's place in the workflow of either a preplanned procedure (e.g. as is done with the Robodoc system), or a cooperative procedure (e.g. as employed in the Rio system), is shown in Figure 2.

We have previously accomplished mastoidectomy using a serial industrial robot $^{6}$ and also using a small bone-attached robot custom designed for mastoidectomy ${ }^{7}$ (see Figure 3 ). The algorithm described in this paper was inspired by these systems but is broadly applicable to all bone milling procedures. It is also worth noting that for simple linear drill trajectories, a planning approach based on probability thresholds like those we use has been previously proposed ${ }^{11}$, which inspired our current work in which we generalize to arbitrarily shaped regions to be milled. The purpose of this paper is to incorporate uncertainty in the position of the robotically controlled drill into path planning for a general path to prevent inadvertent damage to vital anatomy.

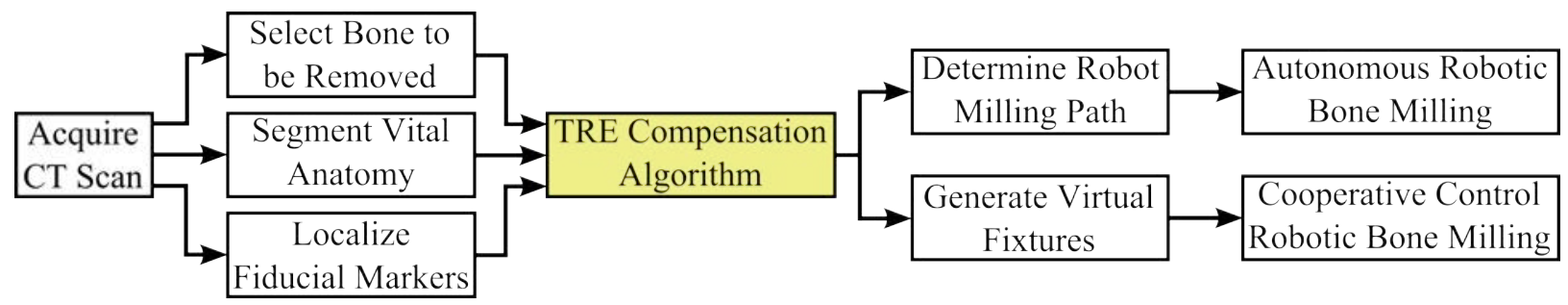

Figure 2: The flowchart illustrates the TRE compensation algorithm's place in the surgical workflow for robotic bone milling. It applies to both procedures where milling is performed autonomously and in procedures that use a cooperative paradigm in which the robot enforces virtual walls to prevent milling of unintended anatomy. 


\section{ALGORITHM DESCRIPTION}

The inputs to our algorithm (see Figure 2) include a preoperative CT scan that is segmented into a Target Region and a series of $N$ Protected Regions, each with an associated maximum allowable probability of damage. The algorithm also requires the locations of the fiducial markers used for registration. Typically the markers will be bone screws that can be
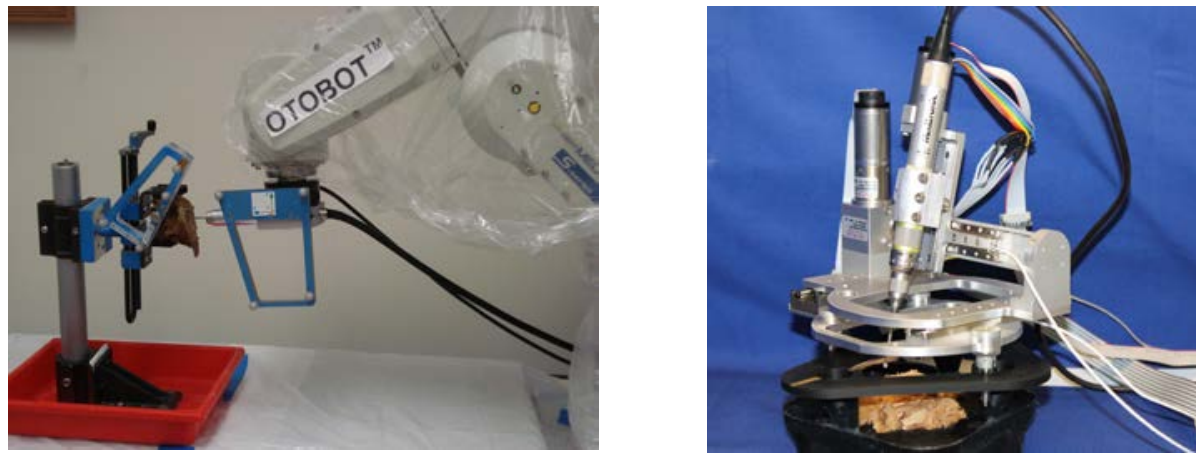

Figure 3: Two examples of robotic platforms we have used to perform mastoidectomies in cadaver studies. A large serial arm is pictured on the left, and a smaller bone-attached 4-axis manipulator is on the right.

identified both in the CT scan and in physical space. The output of the algorithm is a modified Target Region that is slightly smaller than the original because of the presence of a variable thickness safety margin surrounding each Protected Region. The thickness of the safety margin is set by the maximum allowable probability of damage for each Protected Region. The purpose of the algorithm that follows is to define the thickness of this safety margin at all points.

Our algorithm uses voxels that discretize 3D space. It begins with simulated Fiducial Localization Error (FLE), which is generated by injecting Gaussian noise into fiducial positions. The covariance of this Gaussian should be estimated based on experiments with the particular fiducials and imager used $\left(\mathrm{see}^{12}\right)$. In the simulations in this paper, for each of the $\mathrm{x}, \mathrm{y}$, and $\mathrm{z}$ components of fiducial error we sampled an independent Gaussian distribution using the same standard deviation (i.e. the same isotropic FLE for each fiducial) of $0.173 \mathrm{~mm}$ (total magnitude of $0.3 \mathrm{~mm}$ ). This FLE is based on experimental results in $^{12}$ and should be representative of the errors one would see with a portable CT scanner like the Xoran xCAT and spherical stainless steel fiducials. Using these FLE distributions, we generate many possible registrations (in the experiments that follow, this was typically tens of thousands of registrations, but depends on the desired safety probabilities, as discussed later). This provides a large set of $N_{r}$ possible registrations that are consistent with what should be expected based on the FLE of the system, if one were to re-scan and re-register many times.

These registrations are used to establish a safety margin around each Protected Region (See Figure 4). If any of this safety margin extends into the Target Region, the intersection is removed from the Target Region. The purpose of Sections 2.1-2.3 is to describe an iterative process illustrated in Figure 5, which determines the thickness of a given safety margin for one Protected Region based on the surgeon-specified maximum allowable probability of damage to that structure. Section 2.4 describes the procedure needed to apply the results of Sections 2.1-2.3 to multiple Protected Regions simultaneously to generate the final Target Region to be drilled.

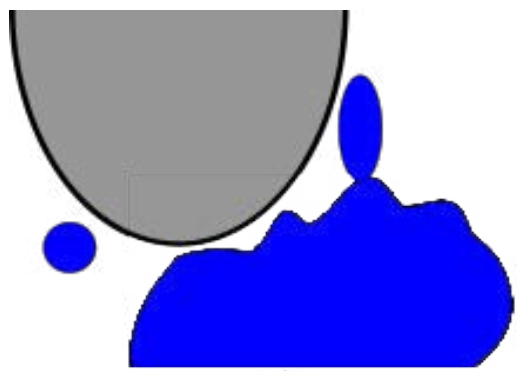

a)

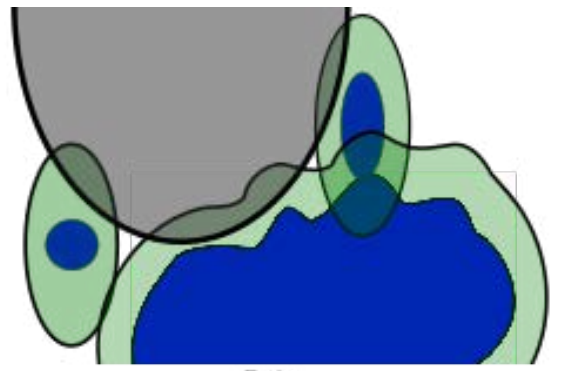

b)

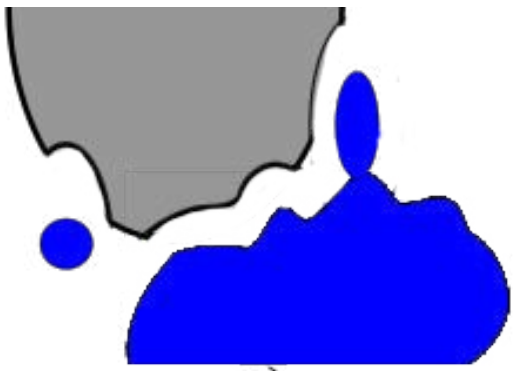

c)

Figure 4: A 2D sample segmentation is shown in a) where blue represents protected regions and gray signifies target to be drilled. Part b) includes green TRE Safety Margins surrounding each of the Protected Regions. Part c) shows the reduced Targeted Region that is the result of our algorithm. 


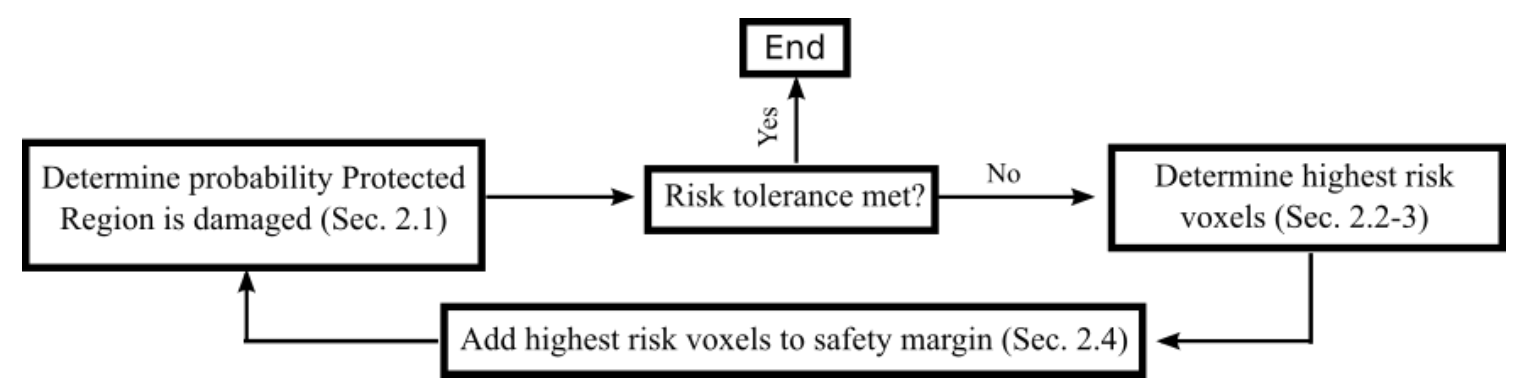

Figure 5: Shown is the procedure followed in the creation of a safety margin for a single Protected Region.

\subsection{Estimating the Probability that a Protected Structure Lies Outside its Safety Margin}

Given a (possibly zero thickness) safety margin surrounding a Protected Region, we determine the probability that the Protected Region extends beyond its safety margin as follows. We use the set of $N_{r}$ possible registrations discussed previously. Applying these to the Protected Region while holding the safety margin fixed, enables one to determine the fraction of registrations for which some part of the Protected Region lies outside the safety margin. That fraction is our estimate of the probability of damage.

\subsection{Determine Highest Risk Voxels}

If the probability calculated in Section 2.1 is greater than desired, we must increase the thickness of the safety margin. To do this, we estimate the probability of damage to the Protected Region if the drill were to drill out each individual voxel surrounding the Protected Region. This is not an easy quantity to estimate in a computationally efficient manner. Naively, one might attempt to create a large set of possible registrations from the FLE as we did previously, but to get statistically significant results requires a large number of possible registrations, for each of a large number of voxels. Also note that to get statistically independent results, one would have to regenerate the set of possible registrations for each voxel considered.

Fortunately, given FLE and the fiducial positions, we can use the results in ${ }^{10}$ to determine the covariance matrix of target registration error at each voxel in a computationally efficient manner. Using these covariance matrices, we calculate a Mahalanobis distance to estimate the risk associated with drilling each voxel. In Section 2.3 we will set a threshold on these risk estimates to determine which voxels to add to the safety margin.

The Mahalanobis distance is useful because it predicts the likelihood that an event occurs within an ellipsoid surrounding a distribution's mean. In our case, the mean is the voxel of interest's nominal location, and the event is the mean plus TRE lying inside the Protected Region. This likelihood can be obtained by evaluating the Chi Squared Cumulative Density Function with three degrees of freedom at the square of the Mahalanobis distance. Based on this, the Mahalanobis distance provides a measure of risk, and we use this risk to rank voxels surrounding the Protected Region from highest to lowest risk. A graphical illustration of the TRE distribution and an ellipsoid illustrating the Mahalanobis distance to an example protected region for the voxel at the centroid of the ellipsoid is shown in Figure 6. 


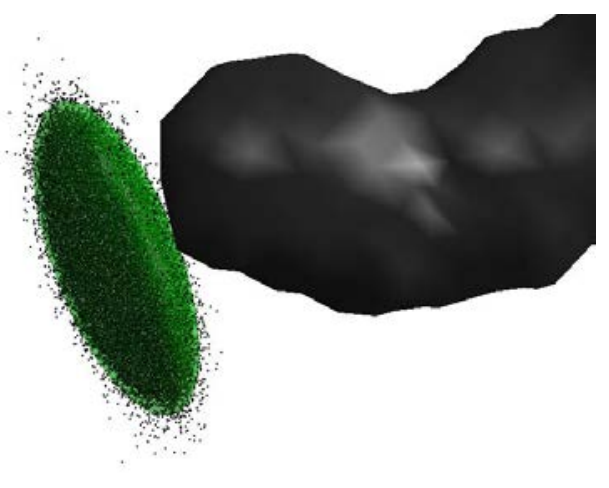

Figure 6: This figure shows an example TRE distribution that has been sampled 500,000 times for the voxel at the centroid of the ellipsoid, which illustrates the minimum Mahalanobis distance to the Protected Region. For the case depicted here, the Mahalanobis distance is 3.65 , which corresponds to a 0.996 probability that the voxel of interest lies within the ellipsoid shown (and therefore outside of the Protected Region).

We note here that this estimate is conservative, since it is possible that the voxel's location including TRE lies outside the ellipsoid and in a direction away from the Protected Region. Note that this will not make the entire algorithm overly conservative, since this ranking will simply be used in Section 2.3 to enlarge the Safety Margin iteratively, yet each time it is enlarged, the overall probability that the Protected Region is damaged is checked using the methods in Section 2.1, which are accurate provided a large number of sample registrations is employed.

\subsection{Update Safety Margin}

Since it would typically be prohibitively expensive computationally to analyze all the voxels in the anatomical region of the surgery, we instead analyze a shell around the union of the Protected Region and current Safety Margin that is onevoxel thick. The Safety Margin will then be enlarged by the highest risk voxels (as computed in Section 2.2), and the procedure iterated, as illustrated in Figure 7. A percentage of the highest risk voxels are then transferred from the shell into the Safety Margin. In determining this percentage there exists a tradeoff between computational speed and the potential for the safety margin to be slightly conservative. If one were to transfer just one voxel at each iteration, for

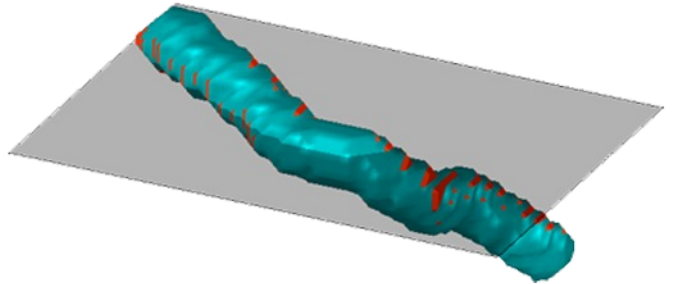

Shell

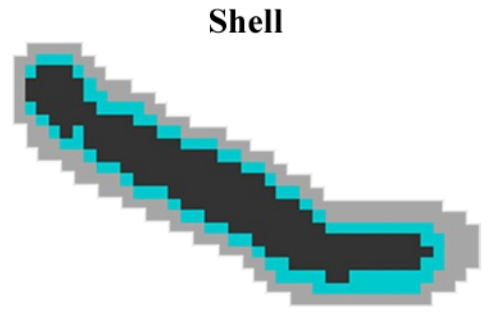

High Risk Voxels

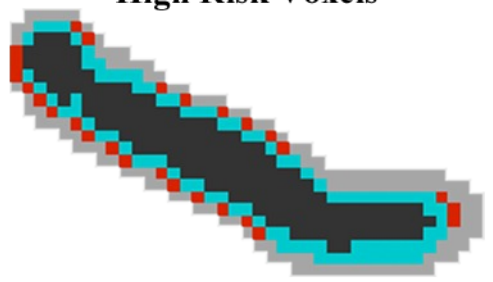

Shell

High Risk Voxels Safety Margin

Protected Region + Safety Margin

Figure 7: An illustration of an example Protected Region and Safety Margin. The bottom three images show the indicated 2D slice, as high-risk voxels are identified and added to the Safety Margin. The left image shows the slice at the beginning of an iteration. The middle image illustrates the highest risk voxels. The right image shows how the Safety Margin has been enlarged to encompass the highest risk voxels (i.e., all red voxels have turned blue). 
example, one would converge on the ideal safety margin, but proceed very slowly (i.e. through many iterations before convergence). In contrast, if one were to choose a large fixed number of voxels to transfer, one would require few iterations, but would overshoot on the last iteration, and thus have a slightly conservatively large safety margin. To address this overshoot, we choose a maximum percentage of the shell to transfer into the Safety Margin. Then we choose a threshold damage probability value after which we linearly reduce the volume transferred into the Safety Margin as the current damage probability approaches the desired damage probability. This behavior is illustrated in Figure 8 .

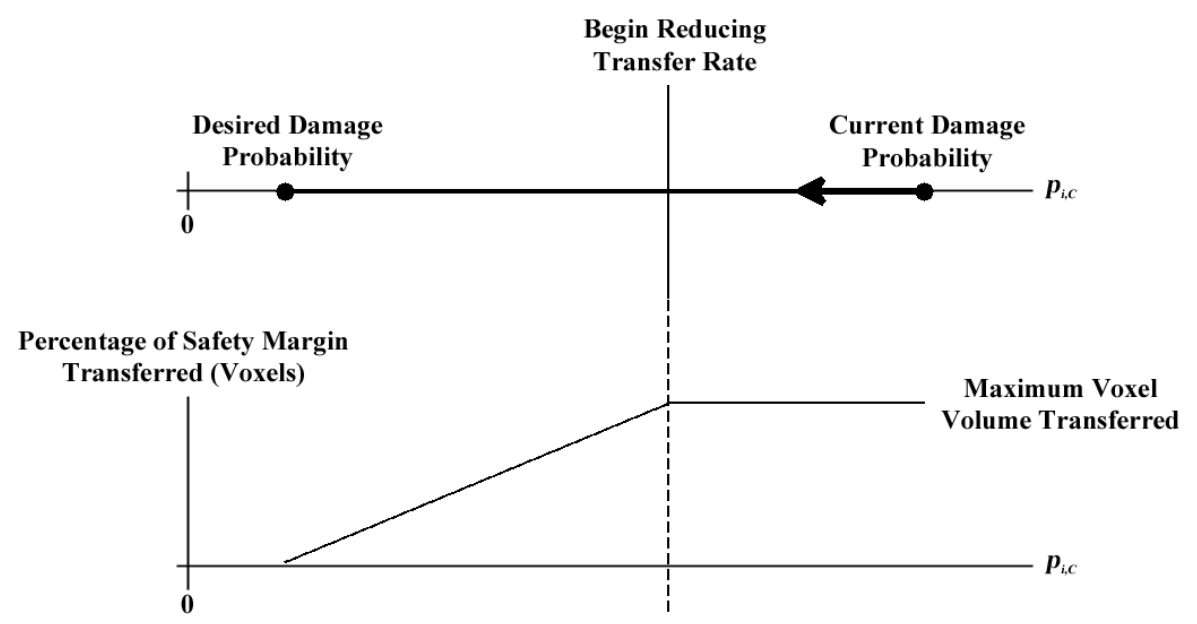

Figure 8: Shown is a visual description of the relationship between the parameters governing the reduction of the quantity of voxels transferred from the shell into the Safety Margin.

\subsection{Algorithm for Reducing Volume to Be Drilled Based on Multiple Protected Regions}

Here we describe the process illustrated in Figure 5, in which we apply the results of Sections 2.1-2.3 to multiple Protected Regions simultaneously and generate the final Target Region to be drilled. The algorithm is as follows:

1. Establish the volume to be drilled by segmenting the CT scan into the Target Region and $i$ Protected Regions, each with its own maximum allowable probability of damage, $p_{i, D}$.

a. Initialize for Protected Region $i$

i. The shell of voxels to be analyzed (all voxels 26-connected to the union of the Protected Region and the Safety Margin)

ii. The Safety Margin as an empty set

b. Simulate registrations with random FLE to estimate the current iteration's probability that the Protected Region is damaged, $p_{i, C}$.

c. Using the TRE distributions associated with each voxel, rank the voxels within the shell by their individual probabilities of damaging structure $i$.

d. Transfer a percentage of the highest risk voxels from the shell to the Safety Margin.

e. Establish new shell ${ }^{\dagger}$

${ }^{\dagger}$ Update shell by including in it all voxels 26-connected with voxels moved in step d that are not in the union of the Protected Region, Safety Margin, and shell. 
f. Repeat steps b-e until $p_{i, C} \leq p_{i, D}$.

g. Take the union of the Safety Margin and the Protected Region to define the volume that must not be drilled for the $i^{\text {th }}$ Protected Region.

2. Repeat step 1 for each of the $N$ protected regions.

3. Subtract the union of the $N$ Augmented Protected Regions from the set of target voxels to be drilled segmented in step 1.

\section{SIMULATION RESULTS}

\subsection{Mastoidectomy}

We applied our approach to a segmented CT scan for a mastoidectomy. The scan dimension is $640 \times 640 \times 355$ with a $0.4 \mathrm{~mm} \times 0.4 \mathrm{~mm} \times 0.4 \mathrm{~mm}$ voxel size, the Facial Nerve and Chorda Tympani were automatically segmented via previously developed methods ${ }^{13}$ and the Target Region was segmented manually by a surgeon. The scan was segmented into targeted bone, Facial Nerve, and Chorda Tympani, as shown in Figure 9. Three fiducials were placed in a triangular pattern $9.4 \mathrm{~mm}$ above the exterior edge of the target region, which represents the approximate attachment location of a bone-attached robot ${ }^{7,14}$ (also see Figure 3). Here, we use the surgeon-specified safety probabilities for various interal ear structures described in ${ }^{11}$, which are $p_{l, D}=0.001$, for the facial nerve, $p_{2, D}=0.2$ for the Chorda Tympani, and $p_{3, D}=0.25$ for other locations on the boundary of the Target Region to be drilled. The parameter from Figure 8 we held constant across the Protected Regions was Maximum Voxel Volume Transferred per iteration $=10 \%$. For the Target Region Boundary and the Chorda Tympani we begin reducing the transfer rate at $p_{i, C}-p_{i, D}=0.4$, and for the Facial Nerve we used $p_{i, C}-p_{i, D}=0.0075$. The FLE used in the simulation is $0.3 \mathrm{~mm}$ each in image space and physical space.
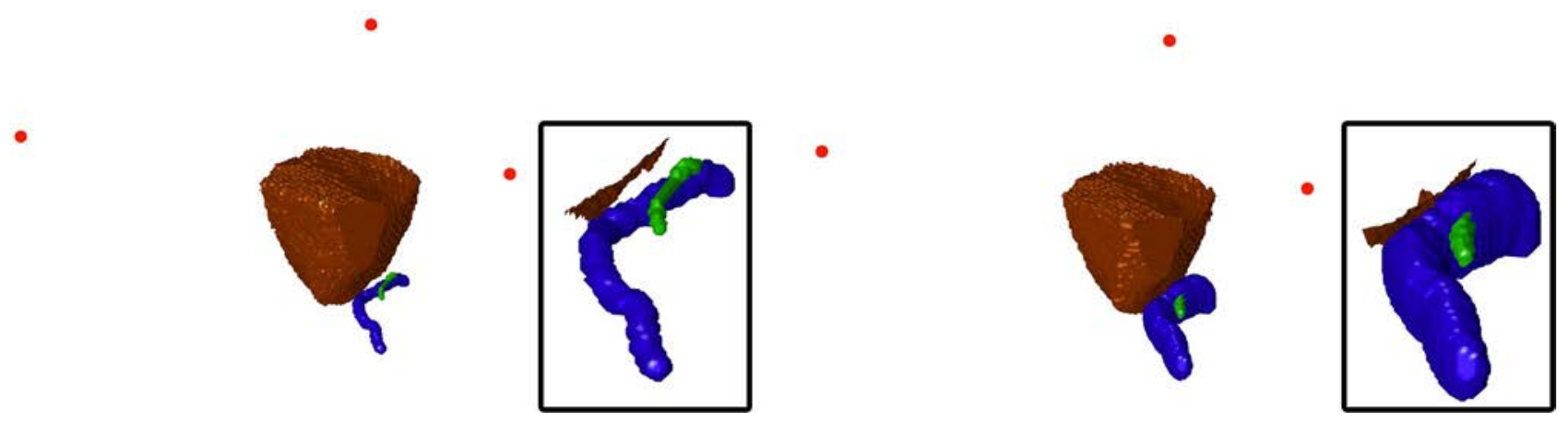

Figure 9: On the left, Facial Nerve (blue), Chorda Tympani (green), Target Region (brown), and fiducials (red) are shown before TRE Safety Buffers are added. On the Right, Safety Margins for Facial Nerve, Chorda Tympani, and Target Region are shown. Overlap of Safety Margins with target voxels to be drilled can be seen in the highlighted window. These overlapping voxels are removed from the set of voxels to be drilled to reduce the probability of damaging nerves to the desired value.

We ran the algorithm on the segmentation shown in Figure 9. The results are shown in the 3 fiducial column of Table 1. The final damage probabilities were 0.00082 for the Facial Nerve, 0.2 for the Chorda Tympani, and 0.25 for the Target Region boundary. The fact that these probabilities are so close to the surgeon specified values illustrates that the interpolation described in Section 2.3 was effective. The algorithm was implemented in MATLAB using a parallel pool of 2 workers and with the Mahalanobis Distance calculation run from a mex file. A MacBook Pro with a $2.8 \mathrm{GHz}$ Intel Core 17 processor and $8 \mathrm{~GB}$ of RAM was used to run the simulation. It required 34 minutes and 37 seconds to complete. Figure 9 shows the extent to which the final Safety Margins intersect the Target Region. Targeted voxels (i.e. voxels to be drilled) within this intersection are removed from the targeted set, reducing the Target Region's volume slightly in order to achieve the surgeon specified safety probabilities, as expected. This final targeted set can be used as input to a path-planner to produce a safe path for robotic bone removal, or to define virtual fixtures for cooperative human-robot milling, as discussed in the introduction. 


\subsection{Effect of the Damage Probability Level on Safety Margin}

To examine the effect of the damage probability on the shape and volume of the Safety Margin we ran the algorithm on a Facial Nerve and recorded the volume of the Safety Margin at varying acceptable damage probabilities. The results are shown in Figure 10.

\begin{tabular}{l|c|c|c}
$\begin{array}{l}\text { Acceptable Damage } \\
\text { Probability: }\end{array}$ & $\mathbf{0 . 1}$ & $\mathbf{0 . 0 1}$ & $\mathbf{0 . 0 0 1}$ \\
\hline $\begin{array}{l}\text { Final Damage } \\
\text { Probability }\end{array}$ & 0.097 & 0.0084 & 0.00083 \\
\hline $\begin{array}{l}\text { Safety Margin } \\
\text { Volume (voxels) }\end{array}$ & 2060 & 4590 & 9710 \\
\hline Color in Figure 7 & Red & Green & Blue
\end{tabular}

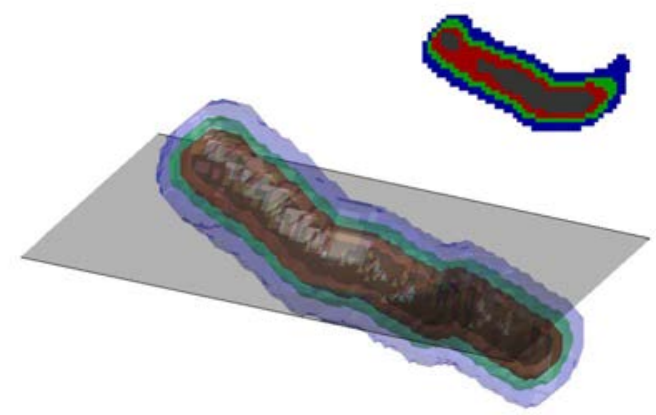

Figure 10: The table shows the volume of the TRE buffer (in voxels) for the fiducial configuration shown in Figure 7 at several different damage probabilities. In the figure on the right a Protected Region is surrounded by three different levels of Safety Margin. These are shown both in 3D and with a 2D slice.

\subsection{Effect of the Number of Fiducial Markers on the Safety Margin}

The robot in Figure 3-right is easily able to accommodate additional fiducial markers. The easiest way to add markers is to place them in a ring around the base of the robot. To investigate the potential benefits of adding additional fiducials in this configuration, we performed the following study. We explored the effects of additional fiducials on the volume of the combined Safety Margin and the Protected Region, investigating use of 3,4,., 12 fiducials, with these fiducials equally spaced around a $50.8 \mathrm{~mm}$ radius circle $9.4 \mathrm{~mm}$ above the Target Region, which is consistent with the robot design shown in Figure 3-right. The results of these simulations are shown in Table 1. It is worth noting that for our mastoidectomy application, the Safety Margin associated with the Chorda Tympani did not require voxels to be removed from the Target Region. Since we could not have known this a priori, it was still valuable to analyze the Chorda Tympani Safety Margin to ensure its safety, despite the fact that it did not cause voxels to be removed in the end.

Table 1: Results for different fiducial configurations.

\begin{tabular}{|c|c|c|c|c|c|c|c|c|c|c|}
\hline Number of Fiducials & 3 & 4 & 5 & 6 & 7 & 8 & 9 & 10 & 11 & 12 \\
\hline \multicolumn{11}{|c|}{ Facial Nerve } \\
\hline $\begin{array}{l}\text { Volume of Safety Margin } \\
\text { (voxels) }\end{array}$ & 9734 & 6683 & 4729 & 4712 & 4597 & 4576 & 4536 & 4282 & 3911 & 3950 \\
\hline $\begin{array}{l}\text { Volume Reduction of } \\
\text { Target Region (voxels) }\end{array}$ & 264 & 93 & 39 & 64 & 59 & 57 & 75 & 70 & 61 & 116 \\
\hline $\begin{array}{l}\text { Percentage Reduction of } \\
\text { Target Region }\end{array}$ & 0.039 & 0.014 & $5 e-3$ & $9.7 e-3$ & $8.9 \mathrm{e}-3$ & $8.6 e-3$ & 0.011 & 0.011 & $9.2 \mathrm{e}-3$ & 0.017 \\
\hline Final Damage Probability & $8.2 \mathrm{e}-4$ & $9.5 \mathrm{e}-4$ & $9.4 \mathrm{e}-4$ & $3.8 \mathrm{e}-4$ & $1.5 \mathrm{e}-4$ & $7.5 \mathrm{e}-4$ & $1.0 \mathrm{e}-4$ & $9.2 \mathrm{e}-4$ & $7.4 \mathrm{e}-4$ & $5.9 \mathrm{e}-4$ \\
\hline \multicolumn{11}{|c|}{ Chorda Tympani } \\
\hline $\begin{array}{l}\text { Volume of Safety Margin } \\
\text { (voxels) }\end{array}$ & 389 & 358 & 336 & 269 & 257 & 250 & 170 & 121 & 2 & 0 \\
\hline $\begin{array}{l}\text { Volume Reduction of } \\
\text { Target Region (voxels) }\end{array}$ & 0 & 0 & 0 & 0 & 0 & 0 & 0 & 0 & 0 & 0 \\
\hline $\begin{array}{l}\text { Percentage Reduction of } \\
\text { Target Region }\end{array}$ & 0 & 0 & 0 & 0 & 0 & 0 & 0 & 0 & 0 & 0 \\
\hline Final Damage Probability & 0.2 & 0.19 & 0.2 & 0.2 & 0.18 & 0.17 & 0.18 & 0.2 & 0.18 & 0.17 \\
\hline
\end{tabular}


Table 1 (continued)

\begin{tabular}{l|c|c|c|c|c|c|c|c|c|c|c|c}
\hline Number of Fiducials & $\mathbf{3}$ & $\mathbf{4}$ & $\mathbf{5}$ & $\mathbf{6}$ & $\mathbf{7}$ & $\mathbf{8}$ & $\mathbf{9}$ & $\mathbf{1 0}$ & $\mathbf{1 1}$ & $\mathbf{1 2}$ \\
\hline \multicolumn{8}{c}{ Target Region Boundary } \\
\hline $\begin{array}{l}\text { Volume of Safety Margin } \\
\text { (voxels) }\end{array}$ & 24348 & 21951 & 20914 & 15971 & 15319 & 13989 & 10519 & 7448 & 3861 & 518 \\
\hline $\begin{array}{l}\text { Volume Reduction of } \\
\text { Target Region (voxels) }\end{array}$ & 24348 & 21951 & 20914 & 15971 & 15319 & 13989 & 10519 & 7448 & 3861 & 518 \\
\hline $\begin{array}{l}\text { Percentage Reduction of } \\
\text { Target Region }\end{array}$ & 3.67 & 3.31 & 3.15 & 2.41 & 2.31 & 2.11 & 1.59 & 1.12 & 0.58 & 0.078 \\
\hline Final Damage Probability & 0.25 & 0.24 & 0.25 & 0.25 & 0.25 & 0.23 & 0.24 & 0.25 & 0.24 & 0.25 \\
\hline
\end{tabular}

The data in this table represents the numerical results of our algorithm applied to several different fiducial configurations in the same cadaver scan. For each case, the fiducials were distributed radially above the target region.

\section{CONCLUSIONS}

We have developed and implemented an algorithm that allows the safety level of any number of protected regions to be specified for general drill motion during bone milling procedures. This algorithm gives the user the ability to set the maximum risks of contact with a set of critical structures to be protected, while ensuring that the robot will mill as much targeted bone as possible. We present simulations using our algorithm for a surgical application that contains several structures of varying criticality, namely a mastoidectomy. From our results it is clear that the more fiducials we include the more certain the registration, and hence fewer targets must be removed from the segmented Target Region to achieve a desired safety probability. These results inform the selection of fiducial configuration balancing the complexity and expense of large fiducial configurations with the volume of bone left undrilled (as required for safety under inaccurate registration).

While we have tested our algorithm in the context of a mastoidectomy, it is applicable to any image-guided procedure reliant on point-based registration where a drill or similar device must pass near structures it can damage but which must be preserved. In the context of bone drilling, this includes both autonomous robot milling of a preplanned volume, and cooperative milling in which the surgeon and robot work together with the robot applying virtual fixtures. Instead of relying on an ad hoc "safety margin", our method replaces heuristics with statistics to provide a principled approach that can give both surgeon and patient confidence in the safety of the procedure.

\section{ACKNOWLEDGMENTS}

The project described was supported by Award Number R01 DC012593 from the National Institute on Deafness and Other Communication Disorders (NIDCD) of the National Institutes of Health (NIH). The content is solely the responsibility of the authors and does not necessarily represent the official views of the NIDCD or NIH.

\section{REFERENCES}

[1] H. A. Paul, W. L. Bargar, B. Mittlestadt, B. Musits, R. H. Taylor, P. Kazanzides, J. Zuhars, B. Williamson, and W. Hanson, "Development of a surgical robot for cementless total hip arthroplasty," Clinical Orthopaedics and related research(285), 57-66 (1992).

[2] S. C. Ho, R. D. Hibberd, and B. L. Davies, "Robot Assisted Knee Surgery - Establishing a Force Control Strategy Incorporating Active Motion Constraint," IEEE Engineering in Medicine and Biology Magazine, 14(3), 292-300 (1995).

[3] J. Abbott, P. Marayong, and A. Okamura, "Haptic Virtual Fixtures for Robot-Assisted Manipulation" Springer Berlin Heidelberg, 5 (2007). 
[4] S. A. Bowyer, Brian L. Davies, and Ferdinando Rodriguez y Baena, "Active constraints/virtual fixtures: A survey," IEEE Transactions on Robotics, 30(1), 20 (2013).

[5] P. A. Federspil, U. W. Geisthoff, D. Henrich, and P. K. Plinkert, "Development of the first force-controlled robot for otoneurosurgery," The Laryngoscope, 113(3), 465-71 (2003).

[6] A. Danilchenko, R. Balachandran, J. L. Toennies, S. Baron, B. Munske, J. M. Fitzpatrick, T. J. Withrow, R. J. Webster, III, and R. F. Labadie, "Robotic mastoidectomy," Otology \& Neurotology, 32(1), 11-6 (2011).

[7] N. P. Dillon, R. Balachandran, A. M. Dit Falisse, G. B. Wanna, R. F. Labadie, T. J. Withrow, J. M. Fitzpatrick, and R. J. Webster, 3rd, "Preliminary Testing of a Compact, Bone-Attached Robot for Otologic Surgery," SPIE Medical Imaging, 9036, 903614 (2014).

[8] T. Xia, C. Baird, G. Jallo, K. Hayes, N. Nakajima, N. Hata, and P. Kazanzides, "An integrated system for planning, navigation and robotic assistance for skull base surgery," The International Journal of Medical Robotics and Computer Assisted Surgery, 4(4), 321-30 (2008).

[9] R. F. Labadie, O. Majdani, and J. M. Fitzpatrick, "Image-guided technique in neurotology," Otolaryngologic Clinics of North America, 40(3), 611-24, x (2007).

[10] A. Danilchenko, and J. M. Fitzpatrick, "General Approach to First-Order Error Prediction in Rigid Point Registration (vol 30, pg 679, 2011),” IEEE Transactions on Medical Imaging, 30(11), 2012-2012 (2011).

[11] J. H. Noble, O. Majdani, R. F. Labadie, B. Dawant, and J. M. Fitzpatrick, "Automatic determination of optimal linear drilling trajectories for cochlear access accounting for drill-positioning error," The International Journal of Medical Robotics and Computer Assisted Surgery, 6(3), 281-90 (2010).

[12] J.-P. Kobler, J. D. Díaz, J. M. Fitzpatrick, G. J. Lexow, O. Majdani, and T. Ortmaier, "Localization accuracy of sphere fiducials in computed tomography images," SPIE Medical Imaging, 90360Z-90360Z-7 (2014).

[13] J. H. Noble, F. M. Warren, R. F. Labadie, and B. M. Dawant, "Automatic segmentation of the facial nerve and chorda tympani in CT images using spatially dependent feature values.," Medical Physics, 35(12), 5375-5384 (2008).

[14] L. B. Kratchman, G. S. Blachon, T. J. Withrow, R. Balachandran, R. F. Labadie, and R. J. Webster, 3rd, "Design of a bone-attached parallel robot for percutaneous cochlear implantation," IEEE Transactions on Biomedical Engineering, 58(10), 2904-10 (2011). 